\title{
Early application of negative pressure wound therapy to acute wounds contaminated with Staphylococcus aureus: An effective approach to preventing biofilm formation
}

\author{
TONGTONG LI ${ }^{1,2}$, LIHAI ZHANG ${ }^{2}$, LI HAN ${ }^{3}$, GUOQI WANG ${ }^{2}$, PENG YIN $^{1,2}$, ZHIRUI LI $^{2}$, \\ LICHENG ZHANG $^{2}$, QI GUO ${ }^{1,2}$, DAOHONG LIU ${ }^{4}$ and PEIFU TANG ${ }^{2}$ \\ ${ }^{1}$ Department of Clinical Medicine, Medical College, Nankai University, Tianjin 300071; \\ ${ }^{2}$ Department of Orthopedics, Chinese PLA General Hospital, Beijing 100853; ${ }^{3}$ Center for Hospital Infection Control, \\ Chinese PLA Institute for Disease Control and Prevention, Beijing 100071; ${ }^{4}$ Department of Orthopedics, \\ The $309^{\text {th }}$ Hospital of PLA, Beijing 100091, P.R. China
}

Received March 23, 2015; Accepted December 16, 2015

DOI: $10.3892 /$ etm.2016.3008

\begin{abstract}
Negative pressure wound therapy (NPWT) has been demonstrated to be effective at preventing biofilm-associated infections; however, its role in biofilm prevention is unknown. The present study evaluated the effect of NPWT on biofilm prevention when rapidly initiated following wound contamination. Full-thickness dermal wounds $(8 \mathrm{~mm})$ were created in rabbit ears and inoculated with green fluorescent protein-labeled Staphylococcus aureus (S. aureus). At $6 \mathrm{~h}$ following inoculation, continuous NPWT at $-125 \mathrm{mmHg}$ was initiated, with the wounds on the contralateral ear left untreated in order to serve as self-controls. $S$. aureus rapidly formed mature biofilms in the wound beds post-inoculation, with a persistent bacterial burden of $\sim 10^{5}-10^{7}$ colony-forming units (CFUs)/wound and impaired wound healing. Compared with the untreated group, NPWT resulted in a significant reduction in biofilm matrix, which was verified by scanning electron microscopy and epifluorescence. A reduction in bacterial counts followed $(\mathrm{P}<0.05)$ with $\sim 10^{3} \mathrm{CFUs}$ /wound on postoperative day 13 and improvement in all healing parameters $(\mathrm{P}<0.05)$ relative to control wounds. The results of the present investigation suggest that NPWT is an effective strategy to impeding the formation of $S$. aureus wound biofilms when initiated rapidly following bacterial contamination. The early application of NPWT, aimed at biofilm prevention, may improve wound care.
\end{abstract}

Correspondence to: Dr Peifu Tang, Department of Orthopedics, Chinese PLA General Hospital, 28 Fuxing Road, Beijing 100853, P.R. China

E-mail: tangpeifu301@sina.cn

Key words: acute wound, bacterial contamination, negative pressure wound therapy, early application, biofilm prevention

\section{Introduction}

Bacterial biofilms have long been considered to be one of the most difficult problems in the field of wound care (1-8). Defined as complex microbial communities irreversibly attached to the wound surface and embedded in self-secreted extracellular polymeric substances (EPS), biofilms provide bacteria with an effective barrier against host immune cells and antimicrobial agents $(6,9)$. As the most common pathogen of wound infection, Staphylococcus aureus (S. aureus) has been widely studied by researchers (10-12). The EPS of staphylococcal biofilms are primarily composed of polysaccharide intercellular adhesin (PIA), extracellular DNA (eDNA), protein and cellular debris $(13,14)$. In particular, PIA and eDNA are the main components of the extracellular matrix of biofilms, and their roles in intercellular adhesion and biofilm formation have been investigated by numerous studies (14-20). Furthermore, the compromised open wounds create an ideal microenvironment for bacterial colonization and biofilm development (6). A series of in vitro and in vivo studies have revealed that wound-associated biofilms grow rapidly and mature within $24 \mathrm{~h}$ post-infection $(12,21-23)$. Once bacteria form a mature biofilm they are difficult to eradicate (8). Despite traditional therapies such as serial debridement and lavage that can remove the majority of mature biofilms and necrotic tissue, residual bacteria may rapidly reestablish a robust biofilm architecture, causing pain to the patients during the process $(24,25)$. The ultimate consequence is a delay in wound healing and reepithelialization $(8,26-28)$.

The durability of a mature biofilm highlights the importance of preventing biofilm formation at the early stages of wound infection. Although specialized dressings for wound care do have an inhibitory effect on biofilms, the efficacy varies greatly with the type, concentration and release of active compound (29). Antibiofilm agents, such as dispersin B and DNase I, have been demonstrated to be effective in the degradation of biofilm matrix both in vitro and in vivo, although these are lacking in clinical application and standard treatment regimens $(9,30)$. In addition, numerous small molecules 
targeting bacterial signaling pathways, such as autoinducing peptide, have demonstrated antibiofilm biological activities, although the clinical efficacy and safety of these compounds has not been sufficiently evaluated $(9,26,31)$. Despite considerable research, a significant improvement in biofilm prevention in the clinical setting remains lacking.

Physical therapies have emerged in biofilm management due to their satisfactory efficacy and low risk for microbial resistance (32). In particular, negative pressure wound therapy (NPWT) has been shown to improve the healing process of infected wounds and to avoid biofilm-associated infections when applied as early as possible (33-38). Previous studies have attributed these benefits to the secondary effects of NPWT, including fluid removal, modulation of inflammation, and the stimulation of wound healing signaling pathways (38-41). However, investigations into the role of NPWT in biofilm formation remain limited. Despite individual studies suggesting its compression effect on established mature biofilms in vitro (42-44), the efficacy of NPWT on biofilm prevention remains unclear, particularly in vivo.

The aim of the present study was to evaluate and validate the potential effect of NPWT on biofilm prevention when initiated rapidly following wound contamination. Subsequent changes in bacterial burden and wound healing secondary to NPWT were also investigated. The results may provide a better understanding of the therapeutic regimen required for wound care.

\section{Materials and methods}

Ethical statement. All animal experiments in the present study were approved by the Medical Ethics Committee of the Chinese PLA General Hospital (Beijing, China) in compliance with the Guidelines for Care and Use of Animals in Research (45).

Animals. A total of 18 adult female Japanese large-ear white rabbits (age, 3-6 months; weight, $\sim 3 \mathrm{~kg}$; purchased from the Laboratory Animal Center of the Academy of Military Medical Sciences, Beijing, China) were used for this study. The rabbits were acclimated to standard housing and fed ad libitum under constant temperature $\left(22^{\circ} \mathrm{C}\right)$ and humidity $(45 \%)$ with a $12-\mathrm{h}$ light/dark cycle.

Bacterial strains and culture. S. aureus strain RN6390 constitutively expressing green fluorescent protein (obtained the from Chinese PLA Institute for Disease Control and Prevention, Beijing, China) was utilized for wound inoculation. S. aureus was grown overnight and subcultured in Luria-Bertani broth (AOBOX Biotechnology Co. Ltd., Beijing, China) at $37^{\circ} \mathrm{C}$ until log phase. Cells were harvested by centrifugation at $4^{\circ} \mathrm{C}$ $(5,000 \times \mathrm{g})$, and washed three times with phosphate-buffered saline (PBS). The final bacterial resuspension was diluted with PBS to an optical density of 1.0 at $600 \mathrm{~nm}$ equivalent to $10^{5}$ colony-forming units (CFUs) $/ \mu 1$ empirically (11).

Wound protocol and bacterial inoculation. The wounding and bacterial inoculation protocol was based on the previously published wound model with minor modifications (12). Briefly, rabbits were anesthetized by intramuscular injection of a ketamine $(45 \mathrm{mg} / \mathrm{kg}$; Gutian Pharma Co., Ltd., Fujian, China) and xylazine (5 mg/kg; Huamu Animal Health Product Co., Ltd., Jilin, China) mixture. Ears were shaved and sterilized twice with $70 \%$ ethanol. Following local anesthesia with $1 \%$ lidocaine (Yimin Pharmaceutical Co., Ltd, Beijing, China), six standardized $8 \mathrm{~mm}$ diameter full-thickness dermal wounds were created by an experienced surgeon on each ventral ear down to the perichondrium using a scalpel. Following hemostasis, the wounds were dressed with semiocclusive IV3000 Transparent Adhesive Film Dressing (Smith \& Nephew Healthcare Ltd., Hull, UK). On postoperative day (POD) 3, each wound was inoculated with $1 \times 10^{6} \mathrm{CFUs}$ of $S$. aureus at a volume of $10 \mu \mathrm{l}$. Planktonic bacteria were allowed to proliferate in vivo under the semiocclusive transparent film for a minimum of $6 \mathrm{~h}$ to ensure bacterial adhesion and colonization $(12,41,46,47)$.

Study design and treatment protocol. All rabbit ears were used to create acute $S$. aureus-contaminated wounds (6 wounds/ear; 36 ears). For each animal, the two ears were respectively and randomly assigned to the 'untreated control group' and the 'NPWT group' (18 ears/group), with the 6 wounds on each ear following the same protocol (32).

The wounds were treated under the previously published protocol with minor modifications (48). Briefly, 6 h postinoculation on POD 3 , the wounds were dressed with a standard NPWT dressing (consisting of polyvinyl alcohol foam, semiocclusive transparent dressing and suction tube; Wuhan VSD Medical Science \& Technology Co., Ltd., Wuhan, China) trimmed in advance to the appropriate size. The suction tube was then connected to a vacuum pump device (provided by Professor Lei Hu, Beihang University, Beijing, China). Wounds treated with NPWT were subjected to continuous negative pressure at $-125 \mathrm{mmHg}$ throughout the study $(41,47)$. Dressings were checked daily and changed on PODs 4, 6, 8 and 10, as recommended for infected wounds by the manufacturer. Animals were sacrificed via an overdose of intravenous pentobarbital sodium $(100-240 \mathrm{mg} / \mathrm{kg}$; Sigma-Aldrich, St. Louis, MI, USA) on PODs $4(\mathrm{n}=8), 6(\mathrm{n}=2)$, $8(n=2), 10(n=2)$ and $13(n=4)$. Wounds were harvested using an 8-mm dermal biopsy punch (Miltex, Inc., York, PA, USA) for PIA, eDNA, viable bacterial count and scanning electron microscopy (SEM) analyses, or a scalpel for epifluorescence and histological analyses.

Detection of PIA and eDNA in wound biofilms. The dorsal side of the wound was removed to eliminate the interference of non-specific bacteria outside of the wound surface (32). The tissue samples were placed in centrifuge tubes with $1 \mathrm{ml} \mathrm{PBS}$, and sonicated for 2 min to remove bacterial biofilms from the tissue $(32,49)$. The insoluble material was discarded by centrifugation at $4^{\circ} \mathrm{C}(13,400 \mathrm{x} \mathrm{g})$. In total, $0.5 \mathrm{ml}$ supernatant was used for the measurement of PIA, and the remainder was used for eDNA extraction. An improved Elson-Morgan assay was performed subsequently to measure the levels of PIA $(13,19,20,50)$. Briefly, $0.5 \mathrm{ml}$ supernatant was supplemented with $0.1 \mathrm{ml}$ potassium tetraborate $(0.8 \mathrm{~mol} / \mathrm{l}$; Vetec; Sigma-Aldrich) in a test tube. Tubes were heated in a boiling water bath for 3 min and were subsequently cooled using tap water. A total of $3 \mathrm{ml}$ Ehrlich's reagent (Vetec; Sigma-Aldrich) 
was added and, immediately after mixing, the tubes were placed in a water bath at $37^{\circ} \mathrm{C}$ for $20 \mathrm{~min}$. The reaction was terminated by cooling the tubes with tap water. The results were expressed as the absorbance of the final reaction solution at $585 \mathrm{~nm}$ as determined using a spectrophotometer (GeneQuant 1300; GE Healthcare Life Sciences, Logan, UT, USA) (50).

The remaining supernatant was used for the extraction of eDNA using the TIANamp Micro DNA kit (Tiangen Biotech Co., Ltd., Beijing, China) according to the manufacturer's protocol. The eDNA levels/wound were expressed as the DNA concentration, quantified using a Qubit ${ }^{\circledR} 2.0$ fluorometer with a Qubit ${ }^{\circledR}$ dsDNA BR Assay kit (both Invitrogen; Thermo Fisher Scientific, Inc., Waltham, MA, USA) according to the manufacturer's protocol (30).

SEM. Following 2.5\% glutaraldehyde and 1\% osmium tetroxide fixation, the tissue samples were dehydrated through a series of graded ethanol $(30,50,70,80,90$ and 100\%) and dried with a critical point dryer (HCP-2; Hitachi, Ltd., Tokyo, Japan) by flooding with liquid carbon dioxide at $5^{\circ} \mathrm{C}$ for $20 \mathrm{~min}$ and raising the temperature to the critical point $\left(\sim 35^{\circ} \mathrm{C}\right)$. Subsequently, samples were mounted using double-sided tape and coated with gold in an auto sputter coater (E-1010; Hitachi, Ltd., Tokyo, Japan). Imaging of the tissue samples was performed by SEM (S-3400N; Hitachi, Ltd., Tokyo, Japan) operated at the scanning voltage of $15 \mathrm{kV}$.

Fluorescent staining and fluorescence light microscopy. The wounds were bisected at the largest diameter and embedded in optimal cutting temperature (O.C.T.) Compound (Sakura Finetek USA, Inc., Torrance, CA, USA), snap-frozen, and stored in liquid nitrogen until cryosectioning. Tissue sections (6 $\mu \mathrm{m})$ were obtained with a Leica CM1950 freezing microtome (Leica Microsystems GmbH, Wetzlar, Germany). Visualization of biofilm matrix was accomplished by staining with Concanavalin A Texas Red ${ }^{\circledR}$ Conjugate (Invitrogen; Thermo Fisher Scientific, Inc.), specific for S. aureus exopolysaccharides, for $15 \mathrm{~min}$ in the dark at room temperature (12). The tissue sections were then rinsed three times in PBS and incubated with 4',6-diamidino-2-phenylindole dilactate (Invitrogen; Thermo Fisher Scientific, Inc.) for 5 min to visualize the host cells. Fluorescence microscopy was performed with an Olympus BX51 microscope (Olympus Corporation, Tokyo, Japan).

Viable bacterial count measurements. Wounds were pretreated as described in the protocol of PIA measurement. Tissue specimens were homogenized into $1 \mathrm{ml}$ suspension with sterile PBS, followed by sonication for $2 \mathrm{~min}$ to disrupt bacterial aggregates in the biofilm. Subsequently, the homogenates were serially diluted with sterile PBS (ranging from $10^{-1}$, $10^{-2}, 10^{-3}$ to $10^{-6}$ times the concentration of the homogenates), plated on Staphylococcus Isolation Agar (Hardy Diagnostics, Santa Maria, CA, USA) and incubated at $37^{\circ} \mathrm{C}$ for $24 \mathrm{~h}$. A standard colony-counting method was conducted and the results were expressed as the logarithm of CFUs/wound $(12,41)$.

Measurement of wound closure. From POD 0 (the day of wounding), images of the wounds were captured with a digital camera (IXUSi; Canon, Inc., Tokyo, Japan) on each day of dressing change. Wound size was then determined by quantifying the wound area using Image-Pro Plus version 6.0 software (Media Cybernetics, Inc., Rockville, MD, USA). The rate of wound closure was expressed as a percentage of the initial wound area (51).

Histological analysis. Wounds were bisected at the maximum diameter and fixed in 10\% neutral formalin (Yili Fine Chemicals Co., Ltd., Beijing, China). The tissue samples were then embedded in paraffin, sectioned into $5-\mu \mathrm{m}$ sections and stained with hematoxylin and eosin. The observation of the tissue sections was performed with an Olympus MVX10 macro-microscope (Olympus Corporation). Images of the stained wounds were captured and analyzed using Image-Pro Plus software, version 6.0. The morphometric parameters included the epithelial and granulation gaps (the distance between the leading edges of newly formed epithelial or granulation tissue) and the epithelial or granulation area (the sum of the area of newly formed epithelial or granulation tissue on the two sides of the wound bed) (28). The measurements were carried out by two independent observers who were blinded to the treatment, and an average result was calculated.

Statistical analysis. Data are reported in graphical form as mean \pm standard deviation. Student's t-test (two-tailed and paired) was used to compare the PIA/eDNA content, viable bacterial counts and histological parameters between NPWT-treated wounds and their untreated control. Wound closure was analyzed using repeated-measures analysis of variance, followed by the least significant difference post-hoc test to evaluate the statistical difference between groups at each time point. All analyses were performed using SPSS software, version 17.0 (SPSS Inc., Chicago, IL, USA) at a significance level of $\alpha=0.05$.

\section{Results}

NPWT reduces PIA and eDNA levels and inhibits the production of extracellular matrix. With the previously established rabbit ear wound model, $S$. aureus-contaminated wounds were treated with or without NPWT from $6 \mathrm{~h}$ postinoculation. For the main components of $S$. aureus biofilm, namely PIA and eDNA, NPWT resulted in a significant reduction in their levels in the wound beds (PIA, P<0.05; eDNA, $\mathrm{P}<0.01$ ) (Fig. 1). This reduction could be visualized through SEM (Fig. 2). The untreated wounds manifested a relatively intact biofilm structure (Fig. 2A) with large amounts of cocci-shaped S. aureus embedded within the lattice-like extracellular matrix (arrows, Fig. 2B). Conversely, wounds treaded with NPWT showed comparable amounts of bacteria but a lack of extracellular matrix (Fig. 2C), which was more visible at a higher magnification (Fig. 2D). To verify the results, epifluorescence microscopy was performed, showing a mature biofilm with large amounts of exopolysaccharide matrix surrounding the bacteria in untreated wounds (Fig. 3A). By comparison, bacteria in the NPWT group spread over the wound beds with sparse exopolysaccharide matrix (Fig. 3B). An uncolonized area of the wound tissue showed background staining of Concanavalin A, and served as a negative control (Fig. 3C). 
A

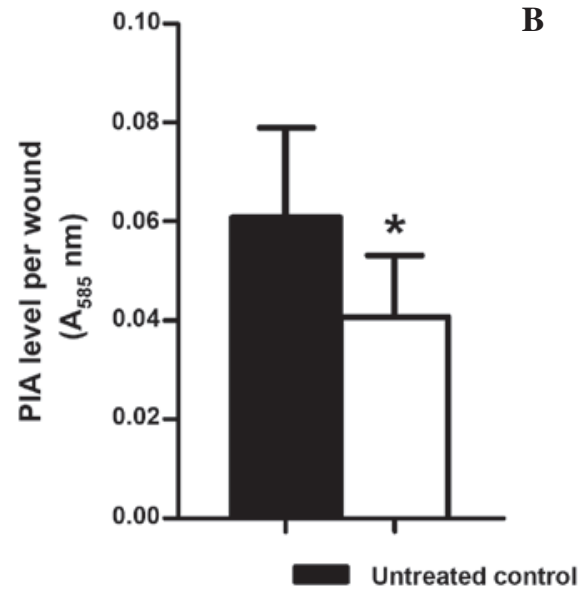

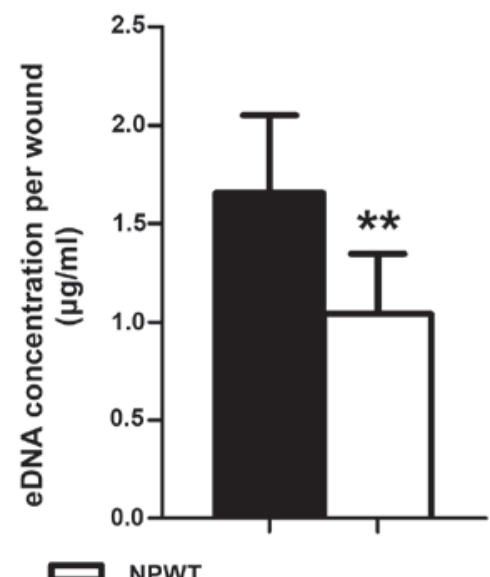

Figure 1. Detection of components of the biofilm matrix from Staphylococcus aureus-infected wounds with and without NPWT treatment. NPWT resulted in significant reductions in (A) PIA and (B) eDNA content relative to untreated wounds. ${ }^{*} \mathrm{P}<0.05$ and ${ }^{* *} \mathrm{P}<0.01$ vs. untreated control. Data are presented as mean \pm standard deviation ( $\mathrm{n}=10-12$ wounds/group). NPWT, negative pressure wound therapy; PIA, polysaccharide intercellular adhesin; eDNA, extracellular DNA; $\mathrm{A}_{585}$, absorbance at $585 \mathrm{~nm}$.

A

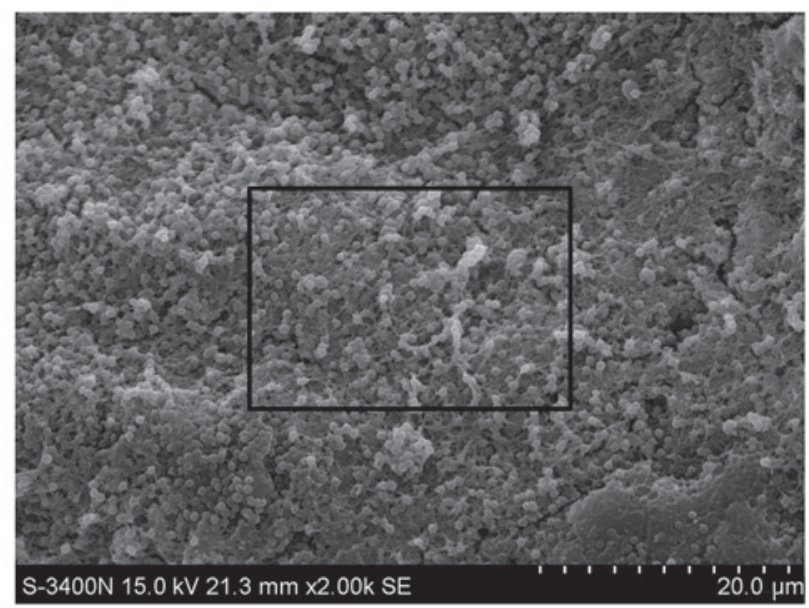

C

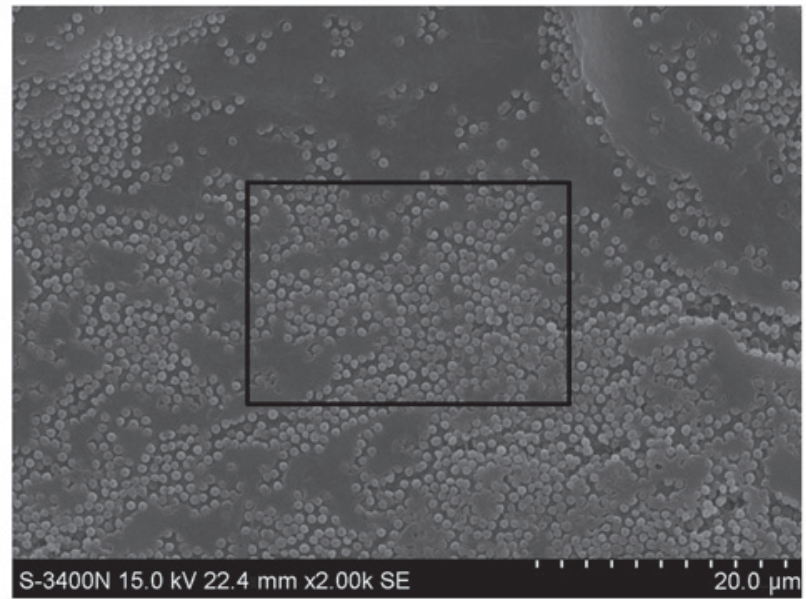

B

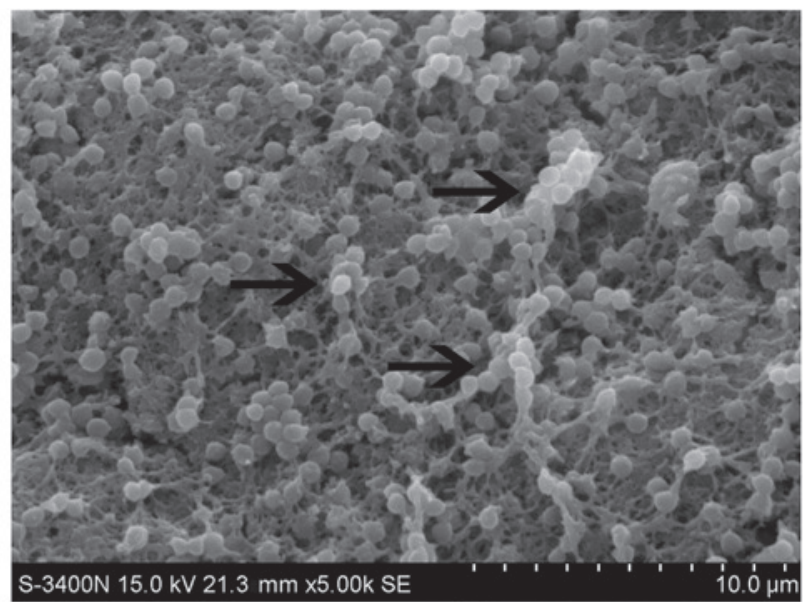

D

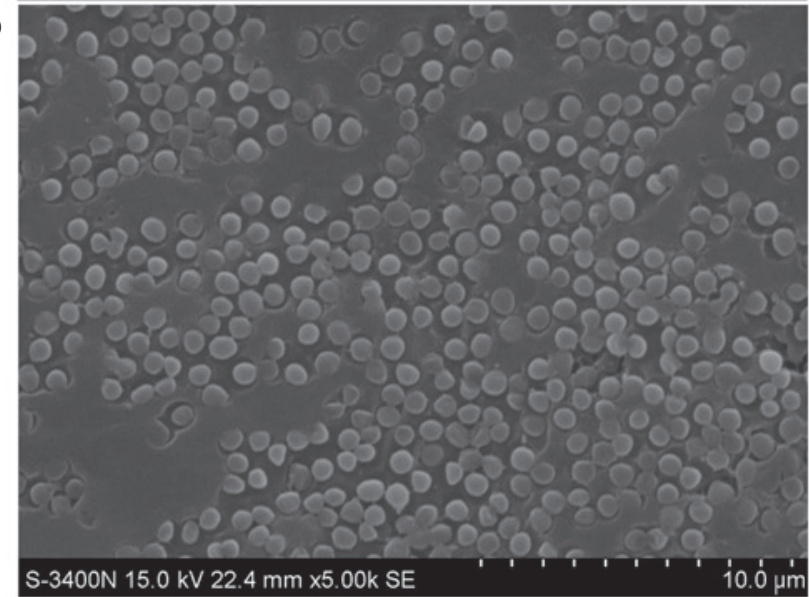

Figure 2. Scanning electron microscopy of Staphylococcus aureus-infected wounds with and without NPWT treatment. (A) The untreated wounds exhibited a mature biofilm structure. (B) Higher magnification of the same visual field showed large amounts of cocci-shaped Staphylococcus aureus embedded within the lattice-like extracellular matrix (arrows). (C) Conversely, wounds treated with NPWT presented approximately equal amounts of bacteria but lack of extracellular matrix, (D) which was more explicit at higher magnification. NPWT, negative pressure wound therapy.

Prolonged NPWT reduces bacterial load. Viable bacterial counts were measured over time to investigate the effect of early application of NPWT on bacterial burden (Fig. 4). On POD 4 and 6, the bacterial load in the wounds treated with NPWT was not significantly different from that in the untreated controls with $\sim 10^{7} \mathrm{CFUs/wound.} \mathrm{However,} \mathrm{from}$ POD 8 there was a significant decline of the bacterial load in the NPWT group (POD 8, P<0.05; POD 10, P<0.01). At the 
A

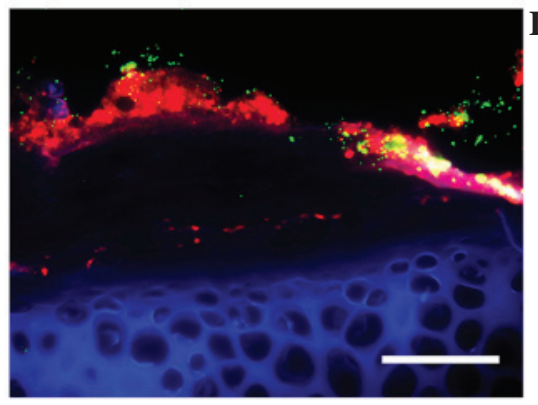

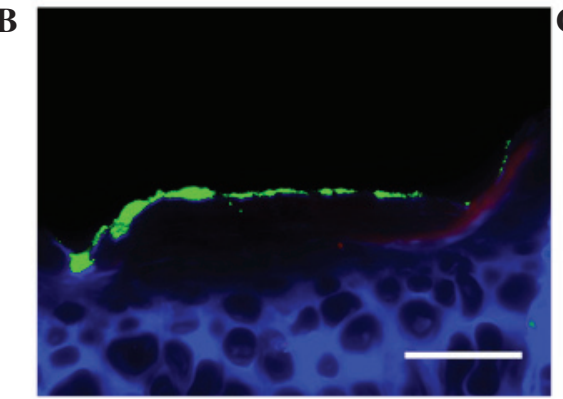

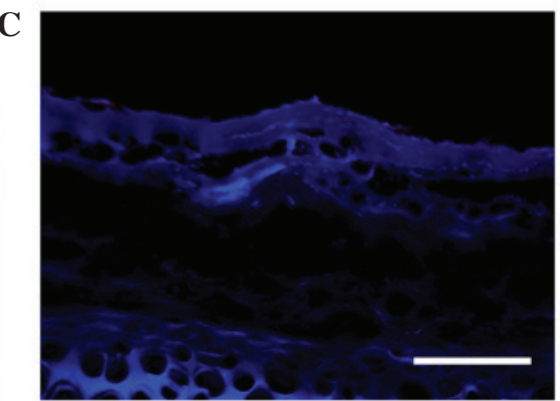

Figure 3. Fluorescence light microscopy of NPWT untreated and treated Staphylococcus aureus-infected wounds counterstained with ConA and DAPI. (A) GFP-labeled Staphylococcus aureus (green) formed a mature biofilm on the untreated wound surface (blue), showing a complex structure with large amounts of exopolysaccharide (red) around the bacteria. (B) Staphylococcus aureus in the NPWT group spread over the wound bed with sparse exopolysaccharide matrix. (C) As a negative control, an area of wound tissue with no bacterial aggregates was shown to exhibit background staining of ConA. Scale $\mathrm{bar}=100 \mu \mathrm{m}$. NPWT, negative pressure wound therapy; ConA, Concanavalin A; DAPI, 4',6-diamidino-2-phenylindole; GFP, green fluorescent protein .

end of the study (POD 13), NPWT resulted in a significant reduction in the bacterial count by two-log fold compared with the untreated wounds $(\mathrm{P}<0.001)$.

NPWT enhances wound healing. The reduction in bacterial burden secondary to NPWT correlated with a synchronous enhancement in wound healing. Gross appearance on POD 13 manifested a marked difference between the two groups. Compared with the film-like exudates overlying the untreated wounds (Fig. 5A), NPWT accelerated wound closure and epithelialization, with clean granulation tissue beds (Fig. 5B). The rate of wound closure in the NPWT group was significantly higher compared with that of the untreated group (Fig. 5C). Images of histological tissue sections showed increased amounts of new epithelial and granulation tissue in NPWT-treated wounds (Fig. 6B) compared with untreated controls (Fig. 6A). Trends in wound healing were quantified by measuring the epithelial and granulation gaps and areas. NPWT led to a significant reduction in epithelial and granulation gaps $(\mathrm{P}<0.001)$ and increase in new epithelial $(\mathrm{P}<0.01)$ and granulation $(\mathrm{P}<0.001)$ areas compared with the untreated wounds (Fig. 6C and D).

\section{Discussion}

Bacterial biofilm remains a challenging issue in the field of wound care (1-8). Although there is a greater understanding of the necessity of biofilm prevention, the therapeutic strategies to prevent biofilm formation are limited in clinical practice $(8,9,24-31)$. In an effort to seek safe and effective wound care modalities, early application of NPWT has been widely acknowledged for its considerable efficacy and low incidence of biofilm-associated infections (32-38). However, despite an increasing number of studies investigating this type of therapy, the potential role of NPWT in biofilm prevention has yet to be elucidated (37-44). The present study examined and evaluated the effect of NPWT on biofilm prevention when initiated rapidly following wound contamination.

The results indicated that the early application of NPWT may be an effective approach for the prevention of biofilm formation in S. aureus-contaminated wounds. Although the exact mechanism underlying the effects of NPWT has yet to be established, a possible explanation for the benefits observed

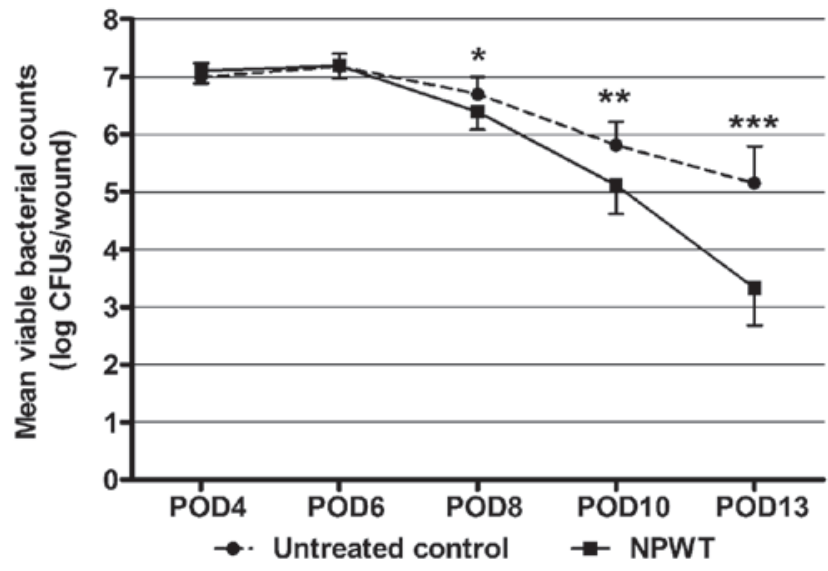

Figure 4. Viable bacterial counts from Staphylococcus aureus-infected wounds with and without NPWT treatment. Serial bacterial counts from untreated wounds revealed a persistent bacterial burden averaging between $10^{5}-10^{7}$ CFUs/wound. However, wounds treated with NPWT showed a gradual but significant reduction in viable bacteria compared with untreated controls, with $\sim 10^{3}$ CFUs/wound on POD 13. ${ }^{*} \mathrm{P}<0.05,{ }^{* *} \mathrm{P}<0.01$ and ${ }^{* * *} \mathrm{P}<0.001$ vs. the untreated control. Data are presented as mean \pm standard deviation ( $\mathrm{n}=10-12$ wounds/group/time-point). NPWT, negative pressure wound therapy; CFUs, colony-forming units; POD, postoperative day.

following treatment with NPWT may be the lack of biofilm matrix. As the structural components of staphylococcal biofilms, PIA and extracellular DNA have an important role in bacterial aggregation and biofilm formation (15-18). Scavenging these matrix components or inhibiting their biosynthesis can effectively prevent biofilm formation and development (9). Previous studies have demonstrated the effectiveness of NPWT in fluid removal and wound cleaning due to the continuous suction (39). The continuous suction may also clear PIA and eDNA from the contaminated wounds before these components are able to form extensive intermolecular crosslinks around the bacteria. In addition, due to the physical effect of negative pressure, NPWT leads to an essentially different microenvironment for wound healing, characterized by hypoxia and microstress (38). These environmental factors, which may stimulate host gene expression and cell proliferation through a variety of signaling pathways $(38,40)$, may also have an effect on the bacteria. Therefore, another possible mechanism is that the environmental factors secondary to NPWT 
A

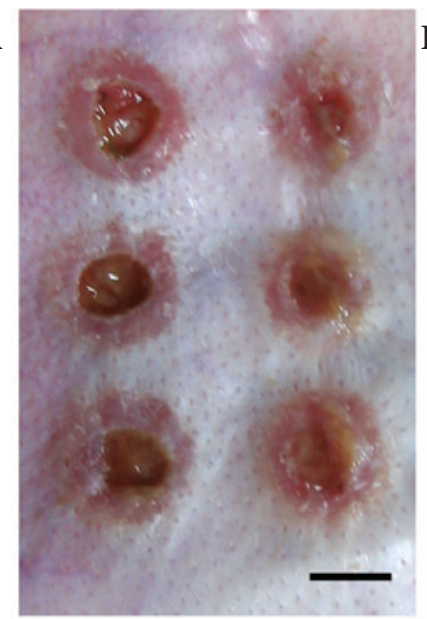

B

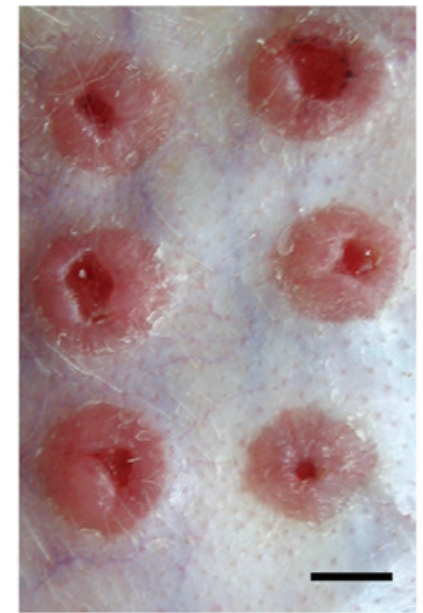

C

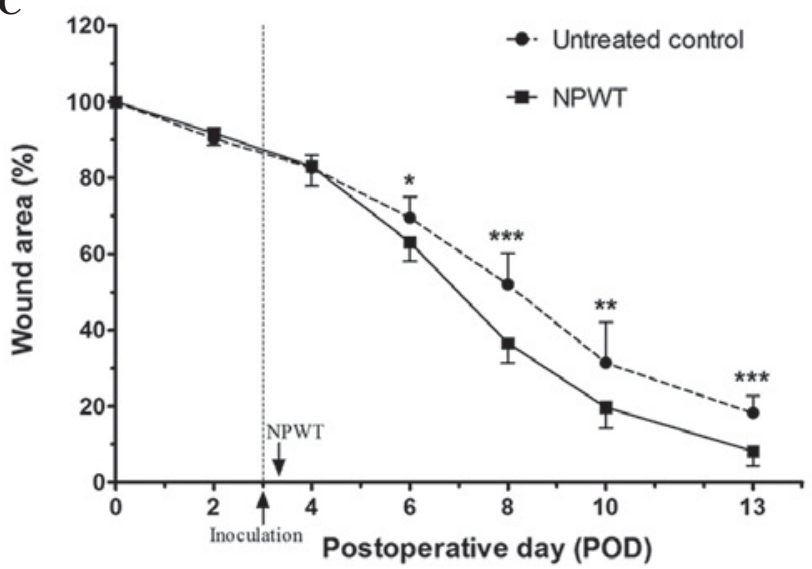

Figure 5. Appearance of wounds and wound closure rates. (A) The untreated wounds on POD 13 manifested a delay in healing and film-like exudates overlying the wound surfaces. (B) Conversely, NPWT accelerated wound closure and epithelialization, with clean granulation tissue beds. (C) The wound closure rate, shown as the percentage of initial wound area, was significantly increased in the NPWT group compared with the untreated control group from POD 6 . (A and B) Scale bar $=5 \mathrm{~mm} .{ }^{*} \mathrm{P}<0.05,{ }^{* *} \mathrm{P}<0.01$ and ${ }^{* * * *} \mathrm{P}<0.001$ vs. the untreated control. Data are presented as mean \pm standard deviation (n=12 wounds/group). NPWT, negative pressure wound therapy; POD, postoperative day.

A

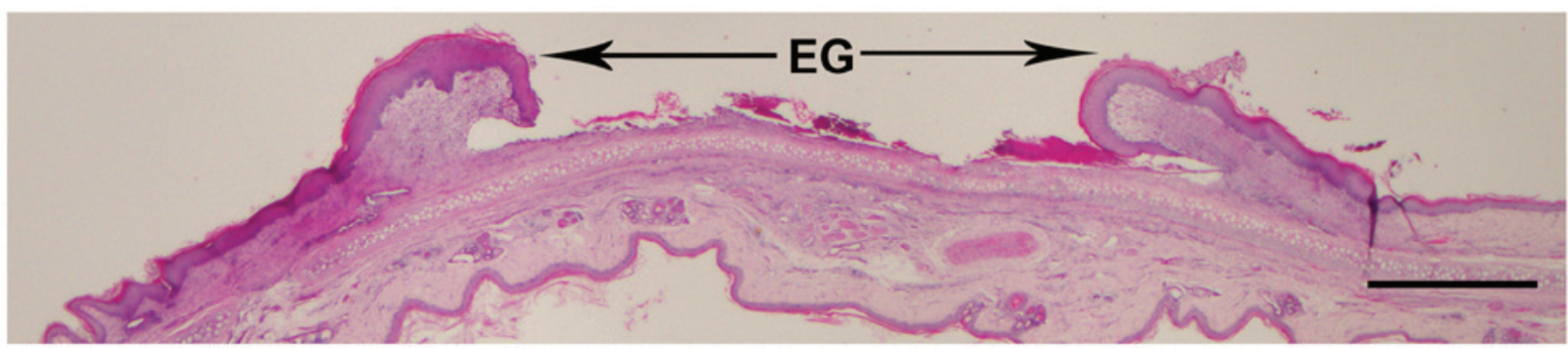

B

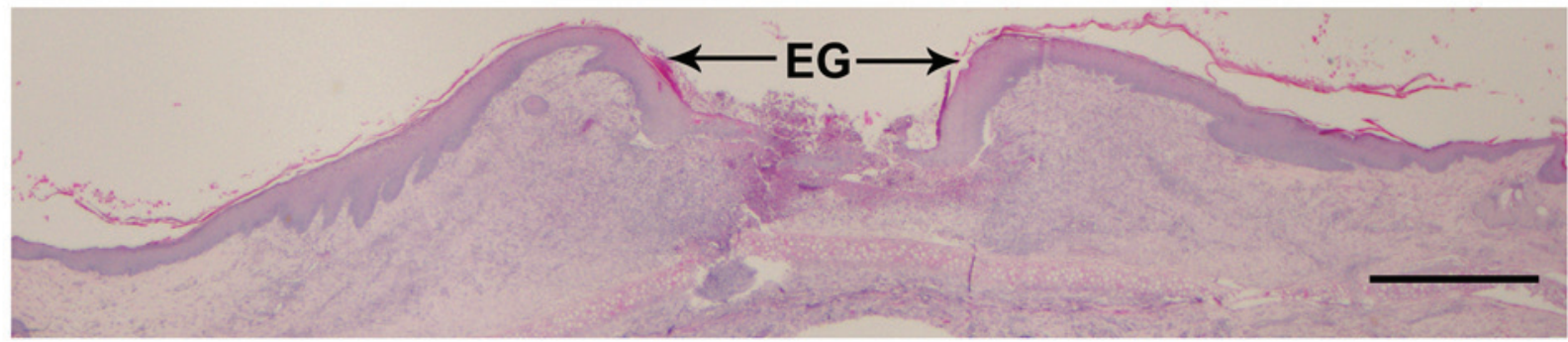

C

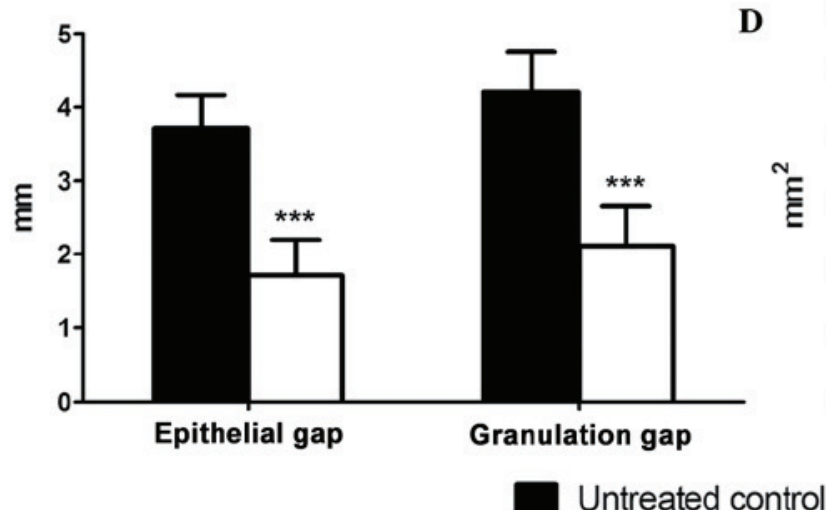

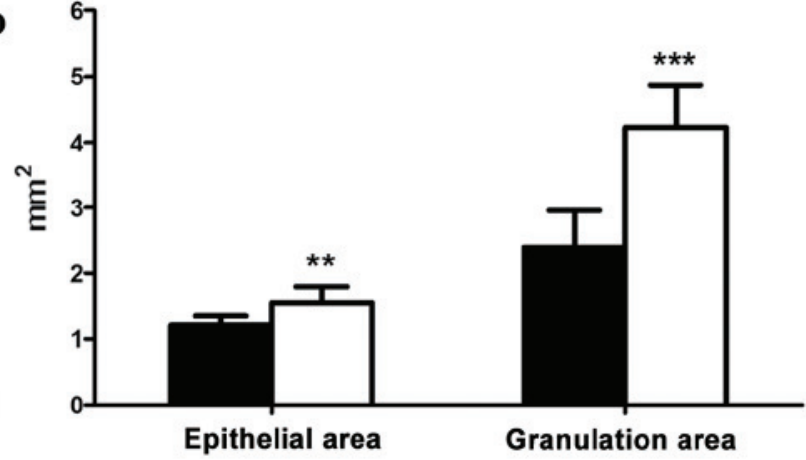

Epithelial area

NPWT

Figure 6. Comparison of histological sections and healing parameters between NPWT untreated and treated Staphylococcus aureus-infected wounds. Histological tissue sections, stained with hematoxylin and eosin on POD 13, showed that compared with (A) the untreated control, the amounts of new epithelial and granulation tissue in (B) the NPWT-treated wounds were increased. As determined by quantitative analysis, NPWT was shown to result in improvements in all healing parameters, (C) with a significant reduction in epithelial and granulation gaps and (D) increase in new epithelial and granulation areas. Scale bar $=1 \mathrm{~mm} .{ }^{* *} \mathrm{P}<0.01$ and ${ }^{* * *} \mathrm{P}<0.001$ vs. the untreated control. Data are presented as mean \pm standard deviation ( $\mathrm{n}=10-12$ wounds/group). EG, epithelial gap; NPWT, negative pressure wound therapy; POD, postoperative day. 
inhibit the biosynthesis of bacterial biofilm components, such as PIA and eDNA. Therefore, the early application of NPWT may prevent biofilm formation in $S$. aureus-contaminated wound beds.

Without the protection of the biofilm matrix, the bacteria may be eliminated by host immune cells more effectively (9), as shown by viable bacterial counts. Notably, the reduction of the bacterial burden should not be attributed to physical suction, as the bacterial counts did not significantly decrease at the early stages of NPWT. The immune response is more likely to have a leading role in bacterial clearance, as previously demonstrated (41,46). Subsequently, a reduction in biofilm matrix and bacterial burden secondary to NPWT may be beneficial to wound healing, since both of these factors are thought to inhibit the healing process (26-28). In addition, NPWT may also improve wound healing through its ability to stimulate host gene expression and cell proliferation (38-40).

Previous studies have investigated the effect of topical negative pressure used with or without bactericide on established mature biofilms in vitro, and demonstrated the presence of several changes in biofilm morphometric parameters and a reduction in bacterial counts (42-44). However, a compression (perhaps fragmentation) effect on biofilm architecture alone was inferred from the results $(42,44)$. There was no evidence that NPWT was able to remove an established mature biofilm. Unlike these previous studies, the present investigation predominantly focused on the initial stages of wound infection, and examined the efficacy of NPWT on biofilm formation and development. For clinicians and researchers, processes that impede biofilm formation at the initial stage have long been regarded as a promising approach to prevent biofilm-associated infections and chronic infections $(25,37)$. Bacteria without the protection of a biofilm matrix are more susceptible to immune cells and antimicrobial treatment $(9,25)$. The results of the present study suggested that the early use of NPWT rapidly following wound contamination may be more effective than delayed application in impeding biofilm formation. These results are concordant with those of previous studies that have demonstrated a satisfactory efficacy of NPWT on managing acute wounds with contamination when initiated early (33-36). Although further investigations are required in order to clarify the role of biofilm matrix reduction in wound care, the present study provides a possible mechanism of action for this therapy.

Despite rigorous methods and significant results, there were limitations to the study. In particular, the effect of NPWT on S.aureus alone was investigated. As the majority of clinical cases are mixed infections, the investigation of other bacterial species is required during further studies to validate the results discussed herein. In addition, compared with previous studies, NPWT was not combined with traditional therapies, such as bactericide or irrigation. Although biofilm formation and development were inhibited by NPWT alone, viable bacterial counts did not decrease until immune cells infiltrated the wounds. Combined treatment is may have the potential to enhance the elimination of bacteria.

In view of the fact that mature biofilms are difficult to eradicate, the demand for effective and practical measures to avoid biofilm-associated infections is rising in clinical settings. Early application of NPWT to wounds contaminated with $S$. aureus has shown a significant efficacy on biofilm prevention in the present study. With an increasing number of clinical trials to validate its role in wound infection management, NPWT appears to reduce the accumulation of biofilm matrix, providing more opportunities for host cells or antimicrobial treatments to eliminate the unprotected bacteria. A better understanding of the potential role of NPWT in wound management and biofilm prevention should contribute to innovations in the field of wound care.

\section{Acknowledgements}

The present study was supported by a grant from the National Natural Science Foundation of China (grant no. 81472112).

\section{References}

1. Costerton JW, Stewart PS and Greenberg EP: Bacterial biofilms: A common cause of persistent infections. Science 284: 1318-1322, 1999.

2. Hall-Stoodley L, Costerton JW and Stoodley P: Bacterial biofilms: From the natural environment to infectious diseases. Nat Rev Microbiol 2: 95-108, 2004.

3. Parsek MR and Singh PK: Bacterial biofilms: An emerging link to disease pathogenesis. Annu Rev Microbiol 57: 677-701, 2003.

4. Edwards R and Harding KG: Bacteria and wound healing. Curr Opin Infect Dis 17: 91-96, 2004.

5. Sen CK, Gordillo GM, Roy S, Kirsner R, Lambert L, Hunt TK, Gottrup F, Gurtner GC and Longaker MT: Human skin wounds: A major and snowballing threat to public health and the economy. Wound Repair Regen 17: 763-771, 2009.

6. Lindsay D and von Holy A: Bacterial biofilms within the clinical setting: What healthcare professionals should know. J Hosp Infect 64: 313-325, 2006

7. James GA, Swogger E, Wolcott R, Pulcini E, Secor P, Sestrich J, Costerton JW and Stewart PS: Biofilms in chronic wounds. Wound Repair Regen 16: 37-44, 2008.

8. Bjarnsholt T, Kirketerp-Møller K, Jensen PØ, Madsen KG, Phipps R, Krogfelt K, Høiby N and Givskov M: Why chronic wounds will not heal: A novel hypothesis. Wound Repair Regen 16: 2-10, 2008.

9. Boles BR and Horswill AR: Staphylococcal biofilm disassembly. Trends Microbiol 19: 449-455, 2011.

10. Moet GJ, Jones RN, Biedenbach DJ, Stilwell MG and Fritsche TR: Contemporary causes of skin and soft tissue infections in North America, Latin America, and Europe: Report from the SENTRY Antimicrobial Surveillance Program (1998-2004). Diagn Microbiol Infect Dis 57: 7-13, 2007.

11. Seth AK, Geringer MR, Nguyen KT, Agnew SP, Dumanian Z, Galiano RD, Leung KP, Mustoe TA and Hong SJ: Bacteriophage therapy for Staphylococcus aureus biofilm-infected wounds: A new approach to chronic wound care. Plast Reconstr Surg 131: 225-234, 2013.

12. Gurjala AN, Geringer MR, Seth AK, Hong SJ, Smeltzer MS, Galiano RD, Leung KP and Mustoe TA: Development of a novel, highly quantitative in vivo model for the study of biofilm-impaired cutaneous wound healing. Wound Repair Regen 19: 400-410, 2011.

13. Sadovskaya I, Vinogradov E, Flahaut S, Kogan G and Jabbouri S: Extracellular carbohydrate-containing polymers of a model biofilm-producing strain, Staphylococcus epidermidis RP62A. Infect Immun 73: 3007-3017, 2005.

14. Otto M: Staphylococcal biofilms. Curr Top Microbiol Immunol 322: 207-228, 2008.

15. Arciola CR, Campoccia D, Speziale P, Montanaro L and Costerton JW: Biofilm formation in Staphylococcus implant infections. A review of molecular mechanisms and implications for biofilm-resistant materials. Biomaterials 33: 5967-5982, 2012.

16. Bayles KW: The biological role of death and lysis in biofilm development. Nat Rev Microbiol 5: 721-726, 2007.

17. Cramton SE, Gerke C, Schnell NF, Nichols WW and Götz F: The intercellular adhesion (ica) locus is present in Staphylococcus aureus and is required for biofilm formation. Infect Immun 67: 5427-5433, 1999. 
18. O'Gara JP: ica and beyond: Biofilm mechanisms and regulation in Staphylococcus epidermidis and Staphylococcus aureus. FEMS Microbiol Lett 270: 179-188, 2007.

19. Wu X, Wang Y and Tao L: Sulfhydryl compounds reduce Staphylococcus aureus biofilm formation by inhibiting PIA biosynthesis. FEMS Microbiol Lett 316: 44-50, 2011.

20. Sadovskaya I, Chaignon P, Kogan G, Chokr A, Vinogradov E and Jabbouri S: Carbohydrate-containing components of biofilms produced in vitro by some staphylococcal strains related to orthopaedic prosthesis infections. FEMS Immunol Med Microbiol 47: 75-82, 2006.

21. Schaber JA, Triffo WJ, Suh SJ, Oliver JW, Hastert MC, Griswold JA, Auer M, Hamood AN and Rumbaugh KP: Pseudomonas aeruginosa forms biofilms in acute infection independent of cell-to-cell signaling. Infect Immun 75: 3715-3721, 2007.

22. Harrison-Balestra C, Cazzaniga AL, Davis SC and Mertz PM: A wound-isolated Pseudomonas aeruginosa grows a biofilm in vitro within 10 hours and is visualized by light microscopy. Dermatol Surg 29: 631-635, 2003.

23. Akiyama H, Huh WK, Yamasaki O, Oono T and Iwatsuki K: Confocal laser scanning microscopic observation of glycocalyx production by Staphylococcus aureus in mouse skin: Does $S$. aureus generally produce a biofilm on damaged skin? Br J Dermatol 147: 879-885, 2002.

24. Seth AK, Geringer MR, Gurjala AN, Hong SJ, Galiano RD, Leung KP and Mustoe TA: Treatment of Pseudomonas aeruginosa biofilm-infected wounds with clinical wound care strategies: A quantitative study using an in vivo rabbit ear model. Plast Reconstr Surg 129: 262e-274e, 2012.

25. Wolcott RD, Rumbaugh KP, James G, Schultz G, Phillips P, Yang Q, Watters C, Stewart PS and Dowd SE: Biofilm maturity studies indicate sharp debridement opens a time-dependent therapeutic window. J Wound Care 19: 320-328, 2010.

26. Schierle CF, De la Garza M, Mustoe TA and Galiano RD Staphylococcal biofilms impair wound healing by delaying reepithelialization in a murine cutaneous wound model. Wound Repair Regen 17: 354-359, 2009.

27. Roche ED, Renick PJ, Tetens SP, Ramsay SJ, Daniels EQ and Carson DL: Increasing the presence of biofilm and healing delay in a porcine model of MRSA-infected wounds. Wound Repair Regen 20: 537-543, 2012.

28. Seth AK, Geringer MR, Galiano RD, Leung KP, Mustoe TA and Hong SJ: Quantitative comparison and analysis of species-specific wound biofilm virulence using an in vivo, rabbit-ear model. J Am Coll Surg 215: 388-399, 2012.

29. Brackman G, De Meyer L, Nelis HJ and Coenye T: Biofilm inhibitory and eradicating activity of wound care products against Staphylococcus aureus and Staphylococcus epidermidis biofilms in an in vitro chronic wound model. J Appl Microbiol 114: 1833-1842, 2013.

30. Watters C, Everett JA, Haley C, Clinton A and Rumbaugh KP: Insulin treatment modulates the host immune system to enhance Pseudomonas aeruginosa wound biofilms. Infect Immun 82: 92-100, 2014

31. Kaplan JB: Biofilm dispersal: Mechanisms, clinical implications, and potential therapeutic uses. J Dent Res 89: 205-218, 2010

32. Seth AK, Nguyen KT, Geringer MR, Hong SJ, Leung KP, Mustoe TA and Galiano RD: Noncontact, low-frequency ultrasound as an effective therapy against Pseudomonas aeruginosa-infected biofilm wounds. Wound Repair Regen 21: 266-274, 2013

33. Costello JP, Amling JK, Emerson DA, Peer SM, Afflu DK, Zurakowski D, Jonas RA and Nath DS: Negative pressure wound therapy for sternal wound infections following congenital heart surgery. J Wound Care 23: 31-36, 2014.

34. Cheng HT, Hsu YC and Wu CI: Risk of infection with delayed wound coverage by using negative-pressure wound therapy in Gustilo Grade IIIB/IIIC open tibial fracture: An evidence-based review. J Plast Reconstr Aesthet Surg 66: 876-878, 2013.
35. Steingrimsson S, Gottfredsson M, Gudmundsdottir I Sjögren J and Gudbjartsson T: Negative-pressure wound therapy for deep sternal wound infections reduces the rate of surgical interventions for early re-infections. Interact Cardiovasc Thorac Surg 15: 406-410, 2012.

36. Anagnostakos K and Mosser P: Negative pressure wound therapy in the management of postoperative infections after musculoskeletal tumour surgery. J Wound Care 23: 191-194, 196-197, 2014

37. Bradley BH and Cunningham M: Biofilms in chronic wounds and the potential role of negative pressure wound therapy: An integrative review. J Wound Ostomy Continence Nurs 40: $143-149,2013$

38. Huang C, Leavitt T, Bayer LR and Orgill DP: Effect of negative pressure wound therapy on wound healing. Curr Probl Surg 51: 301-331, 2014.

39. Orgill DP, Manders EK, Sumpio BE, Lee RC, Attinger CE, Gurtner GC and Ehrlich HP: The mechanisms of action of vacuum assisted closure: More to learn. Surgery 146: 40-51, 2009.

40. Morykwas MJ, Simpson J, Punger K, Argenta A, Kremers L and Argenta J: Vacuum-assisted closure: State of basic research and physiologic foundation. Plast Reconstr Surg 117 (7 Suppl): 121S-126S, 2006

41. Liu D, Zhang L, Li T, Wang G, Du H, Hou H, Han L and Tang P: Negative-pressure wound therapy enhances local inflammatory responses in acute infected soft-tissue wound. Cell Biochem Biophys 70: 539-547, 2014.

42. Ngo QD, Vickery K and Deva AK: The effect of topical negative pressure on wound biofilms using an in vitro wound model. Wound Repair Regen 20: 83-90, 2012.

43. Phillips PL, Yang Q and Schultz GS: The effect of negative pressure wound therapy with periodic instillation using antimicrobial solutions on Pseudomonas aeruginosa biofilm on porcine skin explants. Int Wound J 10 (Suppl 1): 48-55, 2013.

44. Valente PM, Deva A, Ngo Q and Vickery K: The increased killing of biofilms in vitro by combining topical silver dressings with topical negative pressure in chronic wounds. Int Wound J: doi: 10.1111/iwj.12248.

45. Institute of Laboratory Animal Resources (US). Committee on Care, Use of Laboratory Animals, and National Institutes of Health (US). Division of Research Resources: Guide for the care and use of laboratory animals. 8th edition. National Academies Press, Washington, DC, 2011.

46. Lalliss SJ, Stinner DJ, Waterman SM, Branstetter JG, Masini BD and Wenke JC: Negative pressure wound therapy reduces Pseudomonas wound contamination more than Staphylococcus aureus. J Orthop Trauma 24: 598-602, 2010.

47. Assadian O, Assadian A, Stadler M, Diab-Elschahawi M and Kramer A: Bacterial growth kinetic without the influence of the immune system using vacuum-assisted closure dressing with and without negative pressure in an in vitro wound model. Int Wound J 7: 283-289, 2010.

48. Chen SZ, Li J, Li XY and Xu LS: Effects of vacuum-assisted closure on wound microcirculation: An experimental study. Asian J Surg 28: 211-217, 2005.

49. Burian M, Rautenberg M, Kohler T, Fritz M, Krismer B, Unger C, Hoffmann WH, Peschel A, Wolz C and Goerke C: Temporal expression of adhesion factors and activity of global regulators during establishment of Staphylococcus aureus nasal colonization. J Infect Dis 201: 1414-1421, 2010.

50. Reissig JL, Storminger JL and Leloir LF: A modified colorimetric method for the estimation of $\mathrm{N}$-acetylamino sugars. J Biol Chem 217: 959-966, 1955.

51. Lim Y, Levy MA and Bray TM: Dietary supplementation of $\mathrm{N}$-acetylcysteine enhances early inflammatory responses during cutaneous wound healing in protein malnourished mice. J Nutr Biochem 17: 328-336, 2006. 